\title{
SPATIO-TEMPORAL ASSESSMENT OF MIXED LAND-USE IMPACT ON TRAVEL DEMAND
}

\author{
N. Davoudi ${ }^{1, *}$, M. Taleai $^{1}$, M. Molavi ${ }^{1}$ \\ ${ }^{1}$ Faculty of Geomatics, K. N. Toosi University of Technology, Tehran, Iran. (nilufar.davudi224@gmail.com; taleai@kntu.ac.ir)
}

\author{
Commission VI, WG VI/4
}

KEY WORDS: Urban Mobility, Mixed Land-Use, Taxi Trip Data, Spatio-Temporal Analysis, Transportation Planning, Travel Demand

\begin{abstract}
:
Urban mobility pattern studies are one of the interesting issues in GIScience which provide appropriate means for urban transportation planning and management. Mobility across the city has a direct relation with the land-use pattern. This paper investigates the spatio-temporal effect of the land-use mix at street level on urban movement. Taxi pick up and drop-off data in Manhattan was chosen as the sample data in this study. Trips are classified into two parts (weekdays and weekends trips) and then the correlation between mixed land-use and number of trips occurred in different time windows in each street segment, is calculated. Results indicate positive impact and moderate correlation between mixed land-use and number of trips. In streets with high Entropy, homogeneous distribution of the number of trips at each time window for the weekend and non-homogeneous trip distribution for employment and commercial and residential areas for weekday trips was observed. Also, in streets with low Entropy, nonhomogeneous trip distribution at different time windows for both weekday and weekend were observed upon to dominant land-use.
\end{abstract}

\section{INTRODUCTION}

Intra-urban trips occur based on daily activities of the people with a spatio-temporal nature. Accordingly, urban structure and mixed land-use are some of the effective factors in travel demand at different hours of a day. Mixed land-use means a variety of land uses within an area. In an area with mixed landuse, it is expected that many trips will occur (Rupjyoti Bordoloia et al., 2013).

Studies in the field of urban movement modeling have utilized two approaches: using movement data to extract spatial structure of urban properties (Liu. X. et al., 2015), (Long. Y. et al., 2015) and (Zhong. C. et al. 2014), and extracting urban mobility patterns considering environmental parameters and their impact on urban mobility. Changjoo Kim and Shujie Wang by taking into account the household trips in each census tract perceived mixed land-use has no significant impact on travel pattern while it tends to reduce the automobile travels (Changjoo Kim and Shujie Wang, 2015). Petter Næss et al. by investigating influences of residential location on commuting and non-work travel, found that mixed land-use reduces trip distance and automobile travels (Petter Næss et al., 2019). Cervero.R, Radish, C. demonstrated mixed land-use in a street segment will triple walkability for that street segment (Cervero.R, Radish, C., 1996). Diyanah Inani Azmi and Puziah Ahmad by considering three environmental indices such as residential density, mixed land-use and street connectivity found that precincts with high mixed land-use make more walking trips rather than precincts with low mixed land-use (Diyanah Inani Azmi and Puziah Ahmad, 2014).

Land use analysis can be performed at various scales, from site and street to neighborhood and district. The scale is selected based on expected accuracy of application; so scale is an important issue in spatial analysis. Previous researches have considered large scales such as district, census tract or Traffic
Analysis Zone (TAZ), while it may be more appropriate to consider street level as the smallest scale. The aim of this research is to examine mixed land-use spatio-temporal impact on travel demand on street level in Manhattan. Section 2 introduces the research data. Section 3 describes the research methodology and results. The last section presents the conclusion.

\section{DATA}

\subsection{Taxi Trip Data}

2016 Yellow Taxi Trip Data for January has been downloaded from opendata.cityofnewyork.us and used in this study. The sample data includes pick-up and drop-off dates/times, pick-up and drop-off locations and trip distances. A data cleaning process is applied to remove trips with pick-up and drop-off locations $=\left(\begin{array}{ll}0 & 0\end{array}\right)$ and $>13.5$ miles (the realistic longest trips from one side of Manhattan to the other could be around 12 miles). Since travel demand is different on regular days and holidays, trips are classified into two parts: weekdays and weekends. Then the number of trips occurred in each street segment is calculated for three-hour time intervals (Fig. 1\&2).

\footnotetext{
* Corresponding author
} 


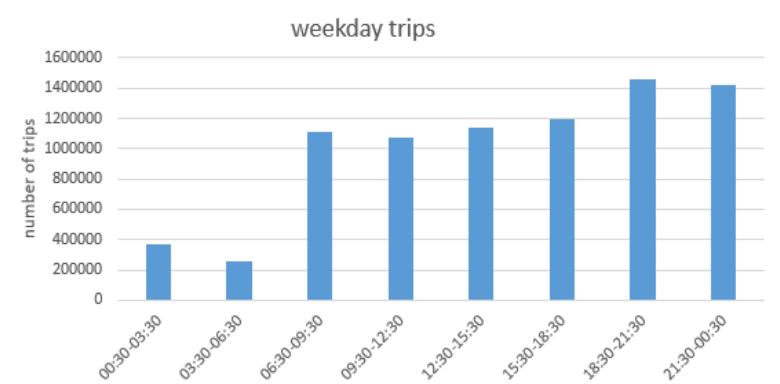

Figure 1. Number of Taxi Trips on Weekdays

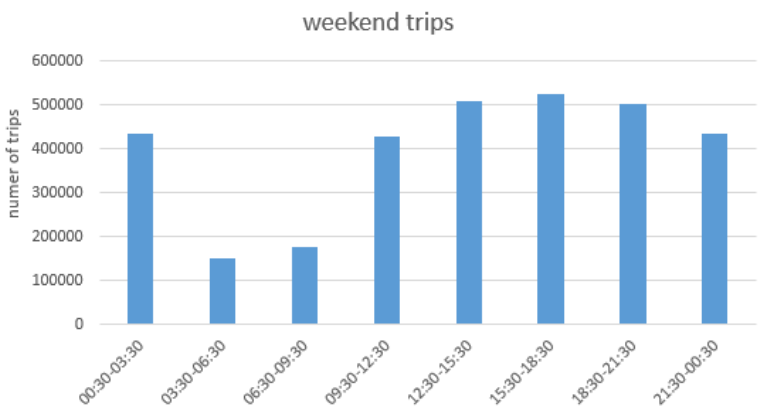

Figure 2. Number of Taxi Trips on Weekends

\subsection{Urban Land-Use Data}

Land use information was extracted from PLUTO data and downloaded from DCP's website. Five land-use categories have been considered: Residential, Employment and Commercial, Recreational, Transportation and Vacant Land. As seen in Fig. 3 most of the employment and commercial places are located in the south of Manhattan and most of the residential places are located in the north.

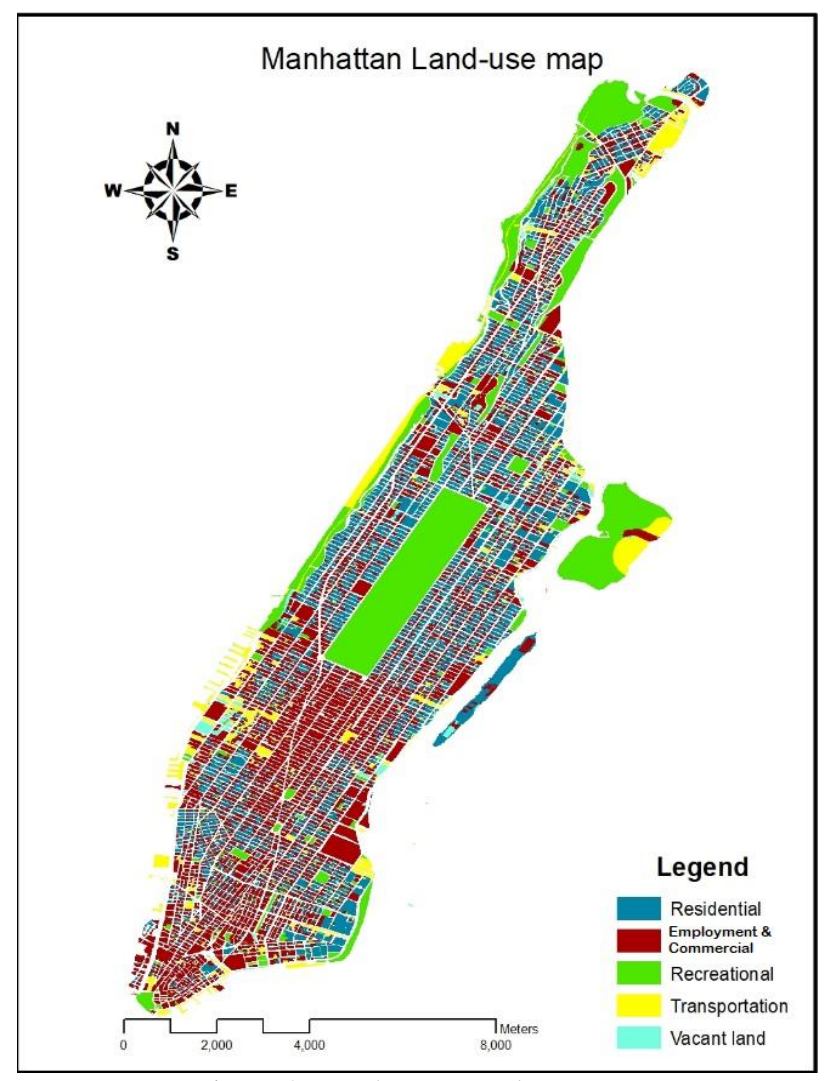

Figure 3. Manhattan Land-use Map

\section{METHODOLOGY}

By assuming that different streets regarding their environmental characteristics might influence the travel demand at different time windows, street-level was chosen as spatial scale in this research. Entropy index as the mixed land-use indicator and the normalized number of trips occurred in each time window as the travel demand indicator have been considered. Then the Spearman correlation coefficient was calculated for these parameters.

In the following of this section, the Entropy Index and Spearman correlation coefficient are explained.

\subsection{Entropy}

There are several methods for quantifying mixed land-use namely Balance Index, HHI, Atkinson, Dissimilarity Index, Gini Index and Entropy (Ghasem Javadi et al 2012). Among these indices, Entropy Index was chosen. This index considers multiple land uses and can measure dispersal and diversity.

Entropy regularly quantifies homogeneity of land use in a given area which is expressed as:

$$
\text { Entropy }=\sum_{j} P_{j} \times \frac{\ln \left(P_{j}\right)}{\ln (J)}
$$

Where $P_{j}$ is the proportion of the total land area of the $j_{t h}$ landuse category found in the tract being analyzed. $\mathrm{J}$ is the total number of land uses considered in the study area (Rupjyoti Bordoloia et al., 2013). The entropy of 0 represents a homogenous land use, and one indicates that the tract of land is equally distributed across all land-use types.

According to Eq.1, Entropy Index has been calculated for each street segment.

\subsection{Correlation Coefficient}

When two variables are discrete or categorical, Spearman rank correlation coefficient is appropriate to assess the correlation between them. Spearman rank correlation coefficient is a nonparametric measure of statistical dependence between two variables. Variable's rank is used instead of the value itself, which is the average of their positions in the ascending order of the values (Wen-Yao Zhang et al., 2016).

$$
r_{s}=\frac{\sum\left\{\left(x_{i}-\bar{x}\right)\left(y_{i}-\bar{y}\right)\right\}}{\sqrt{\sum_{i=1}^{n}\left(x_{i}-\bar{x}\right)^{2} \sum_{i=1}^{n}\left(y_{i}-\bar{y}\right)^{2}}}
$$

Where, $\bar{x}$ and $\bar{y}$ are mean of $X$ and $Y$ traits, $\mathrm{x}_{\mathrm{i}}$ and $y_{i}$ are rank of the measurement of $X$ and $Y$ took on the $i^{\text {th }}$ individual (MarieTherese Puth, et al., 2015).

Spearman correlation coefficient was calculated between Entropy Index of mixed land-use and the number of trips on each street segment occurred in each time window for weekday and weekend trips.

\subsection{Results}

Table 1 shows the Spearman correlation between Entropy Index of mixed land-use and number of trips occurred in different 
time windows for weekdays and weekends. The value of correlations is approximately 0.5 which indicates positive impact and moderate correlation between Entropy and the number of trips in all time windows. It means a mixed land-use impact on travel demand is constant in time variations with an appropriate approximation.

As can be perceived in Manhattan Entropy map, (Fig. 4) Entropy of more than half of the streets is above 0.5 (about $55 \%$ ) which indicates high mixed land-use along with the majority of streets. Entropy and the number of taxi trips occurred in each time window, were investigated for every street and it was found that on weekends, streets with high Entropy have a homogeneous distribution of the number of trips in each time window (Fig. 5). On weekdays, non-homogeneous trip distribution was observed for employment and commercial and residential areas.

Employment and commercial areas at the third and fourth-time windows (6:30-12:30) and residential areas at the fifth and sixth-time windows (12:30-18:30) are exposed to more travel demand and for the remaining of the time windows homogeneous trip distribution was observed (Fig. 6). Also, on streets with low Entropy, non-homogeneous trip distribution was observed depending on dominant land-use, in different time windows for weekday and weekend (Fig. 7).

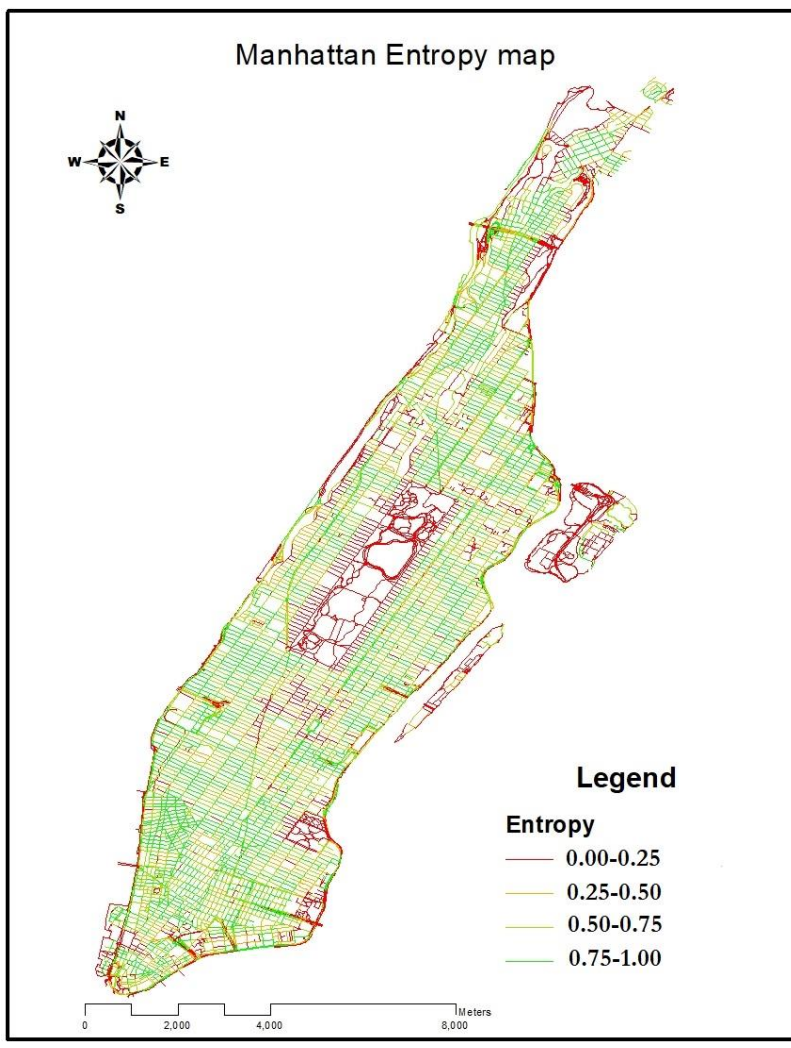

Figure 4. Manhattan Entropy Map

\begin{tabular}{|c|c|c|c|c|c|c|c|c|}
\hline Timewindows & 003010330 & $03: 300.0630$ & $06350-0,30$ & 0930121230 & 123010.1530 & $15: 501-1830$ & 18830202130 & $21: 50-1030$ \\
\hline & 0.528 & 0.500 & 0.488 & 0.484 & 0.491 & 0.499 & 0.520 & 0.522 \\
\hline 8 & 0.536 & 0.516 & 0.481 & 0.489 & 0.488 & 0.496 & 0.515 & 0.56 \\
\hline
\end{tabular}

Table 1. Correlation between Entropy Index and Number of Trips Occurred in Different Time Windows for Weekday and Weekend

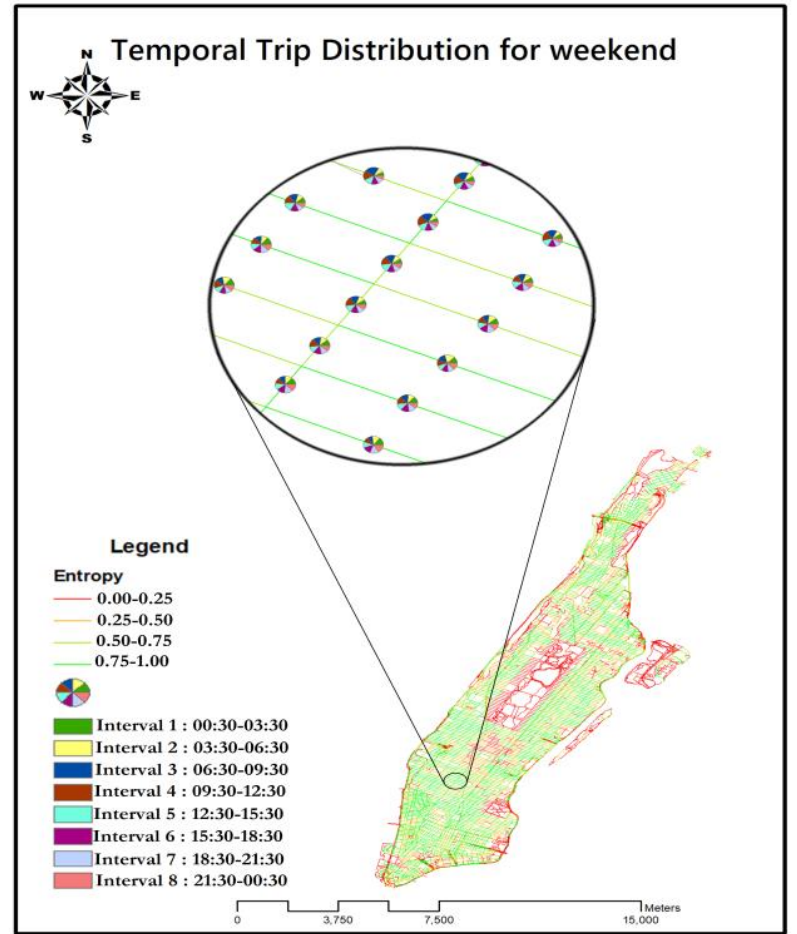

Figure 5. Trip Distribution for High Entropy Streets

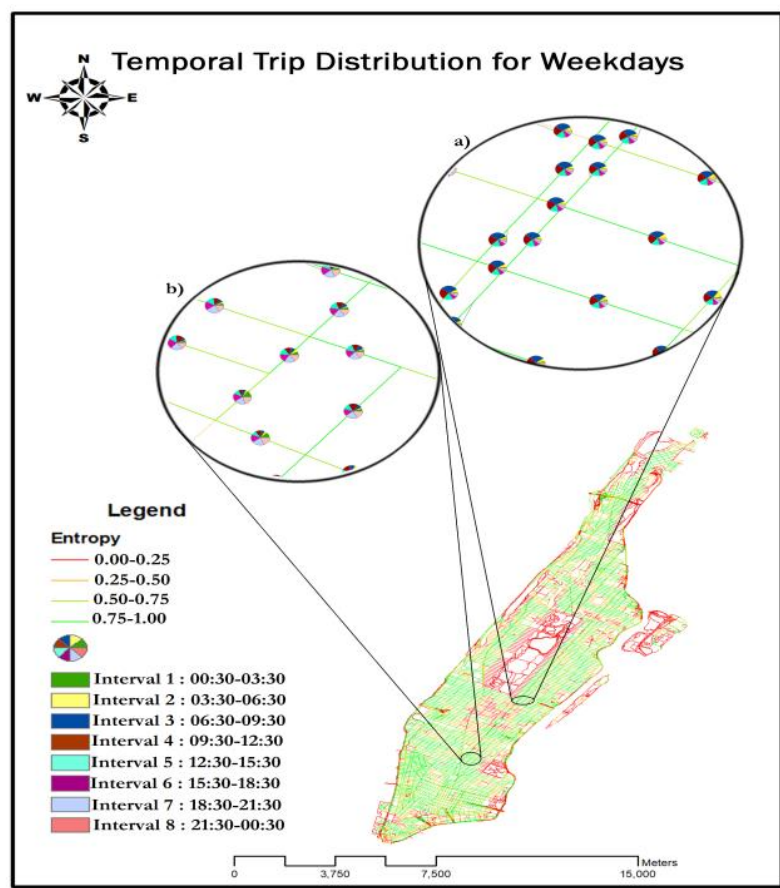

Figure 6. Trip Distribution for a) Employment and Commercial, and b) Residential Areas, in Each Time Window for High Entropy Streets 


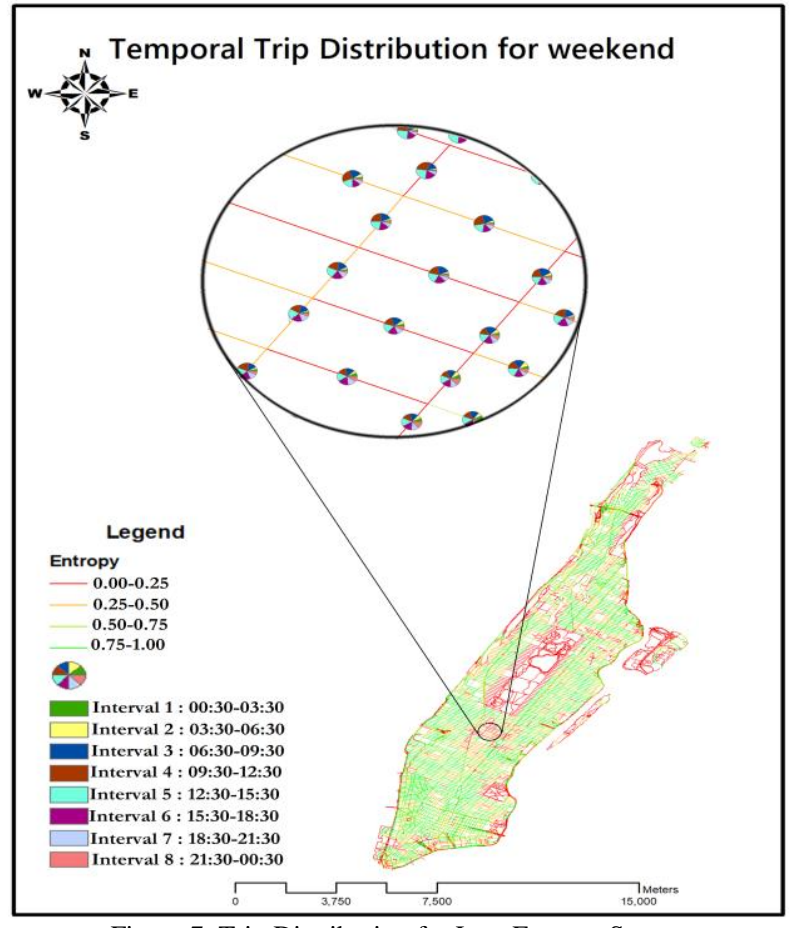

Figure 7. Trip Distribution for Low Entropy Streets

\section{CONCLUSIONS}

In this study, an attempt has been made to evaluate the mixed land-use impact on travel demand in different time windows. Entropy Index was calculated for each street as a mixed landuse index, then, the correlation coefficient between Entropy and number of trips was measured in different time windows for both weekday and weekend trips. The result indicates a positive impact and moderate correlation between Entropy and the number of trips in all time windows. By scrutinizing maps, the following conclusions were drawn:

For streets with high Entropy:

- Homogeneous distribution of the number of trips was observed at each time window for weekend trips.

- Non-homogeneous trip distribution was observed for employment and commercial and residential areas for weekday trips.

and also for streets with low Entropy

- Non-homogeneous trip distribution was observed upon to dominant land-use, at different time windows for weekday and weekend.

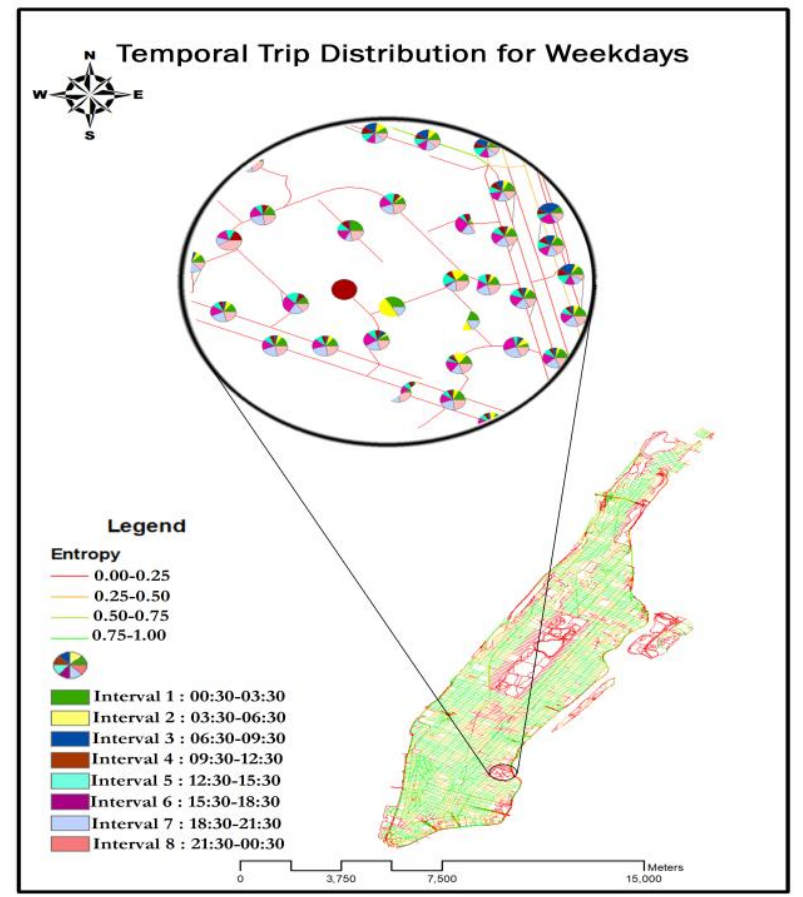

Figure 8. Trip Distribution for Low Entropy Streets

\section{REFERENCES}

Azmi, D.I., Ahmad, P., 2015. A GIS Approach: Determinant of neighbourhood environment indices in influencing walkability between two precincts in Putrajaya. Procedia-Social and Behavioral Sciences, 170, pp.557-566.

Bordoloi, R., Mote, A., Sarkar, P.P. and Mallikarjuna, C., 2013. Quantification of land use diversity in the context of mixed land use. Procedia-Social and Behavioral Sciences, 104, pp.563-572.

Cervero, R., Radisch, C., 1996. Travel choices in pedestrian versus automobile oriented neighborhoods. Transport Policy, 3(3), pp.127-141.

Kim, C., Wang, S., 2015. Empirical examination of neighborhood context of individual travel behaviors. Applied Geography, 60, pp.230-239.

Javadi, G., Taleai, M., Karimi, M., 2013. Development of a Model to Evaluate the Effects of Urban Mixed Land Uses on the Basis of the Spatial Indicators and Analysis. Geography and Territorial Spatial Arrangement. 3(8), pp. 69-84.

Liu, X., Gong, L., Gong, Y., Liu, Y., 2015. Revealing travel patterns and city structure with taxi trip data. Journal of Transport Geography, 43, pp.78-90.

Long, Y., Han, H., Tu, Y., Shu, X., 2015. Evaluating the effectiveness of urban growth boundaries using human mobility and activity records. Cities, 46, pp.76-84.

Næss, P., Strand, A., Wolday, F., Stefansdottir, H., 2017. Residential location, commuting and non-work travel in two urban areas of different size and with different center structures. Progress in Planning. 128, pp.1-36

Puth, M.T., Neuhäuser, M., Ruxton, G.D., 2015. Effective use of Spearman's and Kendall's correlation coefficients for association between two measured traits. Animal Behaviour, 102, pp.77-84. 
Zhang, W.Y., Wei, Z.W., Wang, B.H. and Han, X.P., 2016. Measuring mixing patterns in complex networks by Spearman rank correlation coefficient. Physica A: Statistical Mechanics and its Applications, 451, pp.440-450.

Zhong, C., Arisona, S.M., Huang, X., Batty, M. and Schmitt, G., 2014. Detecting the dynamics of urban structure through spatial network analysis. International Journal of Geographical Information Science, 28(11), pp.2178-2199. 